\title{
Glutamatergic and resting-state functional connectivity correlates of severity in major depression - the role of pregenual anterior cingulate cortex and anterior insula
}

\section{Dorothea I. Horn', Chunshui Yu², Johann Steiner', Julia Buchmann', Joern Kaufmann³, Annemarie Osoba', Ulf Eckert ${ }^{1}$, Kathrin C.Zierhut ${ }^{1}$, Kolja Schiltz' ${ }^{1}$, Huiguang He ${ }^{4}$, Bharat Biswal ${ }^{5}$, Bernhard Bogerts ${ }^{1}$ and Martin Walter ${ }^{1 *}$}

1 Department of Psychiatry, Otto-von-Guericke University, Magdeburg, Germany

${ }^{2}$ Department of Radiology, Tianjin Medical University General Hospital, Tianjin, China

${ }^{3}$ Department of Neurology, Otto-von-Guericke University, Magdeburg, Germany

${ }^{4}$ Key Lab of Complex Systems and Intelligent Science, Institute of Automation, Chinese Academy of Science, Beijing, China

${ }^{5}$ Department of Radiology, New Jersey Medical School, University of Medicine and Dentistry New Jersey, Newark, NJ, USA

\section{Edited by:}

Lucina Q. Uddin, Stanford University, USA

\section{Reviewed by:}

Anke Henning, Institute for Biomedical Engineering, Switzerland

Laura M. Rowland, University of Maryland School of Medicine, USA Alexander Sartorius, Zentralinstitut für Seelische Gesundheit, Germany

\section{${ }^{*}$ Correspondence:}

Martin Walter, CANLAB, Department of Psychiatry, Leipziger Str. 44, 39120 Magdeburg, Germany.

e-mail:martin@canlab.de
Glutamatergic mechanisms and resting-state functional connectivity alterations have been recently described as factors contributing to major depressive disorder (MDD). Furthermore, the pregenual anterior cingulate cortex (pgACC) seems to play an important role for major depressive symptoms such as anhedonia and impaired emotion processing. We investigated 22 MDD patients and 22 healthy subjects using a combined magnetic resonance spectroscopy (MRS) and resting-state functional magnetic resonance imaging (fMRI) approach. Severity of depression was rated using the 21-item Hamilton depression scale (HAMD) and patients were divided into severely and mildly depressed subgroups according to HAMD scores. Because of their hypothesized role in depression we investigated the functional connectivity between pgACC and left anterior insular cortex (Al). The sum of Glutamate and Glutamine (GIx) in the pgACC, but not in left Al, predicted the resting-state functional connectivity between the two regions exclusively in depressed patients. Furthermore, functional connectivity between these regions was significantly altered in the subgroup of severely depressed patients (HAMD > 15) compared to healthy subjects and mildly depressed patients. Similarly the GIx ratios, relative to Creatine, in the pgACC were lowest in severely depressed patients. These findings support the involvement of glutamatergic mechanisms in severe MDD which are related to the functional connectivity between pgACC and $\mathrm{Al}$ and depression severity.

Keywords: major depressive disorder, pregenual anterior cingulate cortex, anterior insula, functional magnetic resonance imaging, glutamate, magnetic resonance spectroscopy, functional connectivity, resting state

\section{INTRODUCTION}

Major depressive disorder (MDD) is characterized by persistent negative feelings of sadness, guilt, and worthlessness and further by ruminating thoughts, cognitive impairments, and somatic complaints. These various symptoms are accompanied by abnormal activity in several brain regions as observed in a number of imaging studies. This supports the notion of a complex network underlying the pathology of depression. It also questions the existence of a few core regions with primarily impaired brain function. Such specific regions, however, would be highly useful to guide regional invasive therapies such as deep brain stimulation (Mayberg et al., 2005; Lozano et al., 2008) or imaging guided treatment prediction or monitoring (MacQueen, 2009).

The medial prefrontal cortex and the anterior cingulate cortex (ACC) have been identified as key structures for MDD, with abnormalities not only in fMRI activation but also in baseline metabolism or perfusion. In accordance with these in vivo findings, post mortem investigations in MDD have revealed reductions in neuronal and glial cell densities, size, and arborization (Ongür et al., 1998; Cotter et al., 2001; Manji et al., 2001). On a molecular level, reduced expression of glial enzymes allowing for glutamate (Glu) reuptake from the synaptic cleft and its conversion to non-excitotoxic glutamine have been observed (Choudary et al., 2005). During the past few years, the hypothesis of a complex metabolic abnormality in the ACC has been developed which links psychological abnormalities in MDD to abnormal baseline metabolism in astrocytes and neurons and even to disease related immunological processes in microglia (Dantzer et al., 2008).

Proton NMR spectroscopy (MRS) is a widely used tool to measure brain metabolites in vivo. MRS enables the detection of metabolites, among them, the total concentration of Glutamate in the brain tissue. A continuous recycling of glutamate between neurons and glial cells takes place in the brain. As neurotransmission progresses, neuronal glutamate is released into the synaptic cleft. From there it is taken up by glial cells and converted to glutamine. Glutamine is afterwards transported back into the neurons where it is reconverted to glutamate (Rothman et al., 2003). Glutamate levels do not only reflect glutamatergic neurotransmitter activity, but also the synthesis of glutamate through the tricarboxylic acid (TCA) cycle. Total glutamate measurements as observed by MRS therefore are not specific to the constant flux through the glutamate-glutamine 
cycle. Glutamatergic alterations in several brain regions have been implicated in neurological or psychiatric diseases using MRS (Auer et al., 2000; Hasler et al., 2007).

In a previous study (Walter et al., 2009), it has been shown that impairments of the neuronal-glial unit can be visualized both on a functional and a molecular level using a combination of $\mathrm{fMRI}$ and MRS. In the pregenual portion of the ACC (pgACC) abnormally reduced amplitudes of negative BOLD responses were found in a group of unmedicated MDD patients. Reduced BOLD amplitudes during task were further related to abnormal glutamate concentrations in the same region. This finding is compatible with the hypothesis that the extent of functional responses that appear on top of baseline neuronal activity depends on the metabolic baseline level of the involved neuronal-astroglial unit and the degree of anaerobic glucose consumption (Raichle and Mintun, 2006). In this first combined resting-state fMRI-MRS study in MDD, we extend previous work (Walter et al., 2009) that relates the amplitudes of negative BOLD responses in pgACC to regional GABA (gammaamino butyric acid) ratios. Interestingly, glutamatergic modulations of BOLD responses were only present in depressed patients, while the GABAergic modulations seen in healthy controls (Northoff et al., 2007) disappeared. A glutamatergic but not GABAergic deficit in pgACC was also reported by Hasler et al. (2007).

A newly arising interrelation of functional responses with a metabolic marker that includes glial functioning would be well in accordance with converging findings in rodents, where pharmacological astroglial disruptions consequently lead to anhedonic behavior such as reduced sucrose preference (Banasr and Duman, 2008). Additionally, agents like riluzole that manipulate the neurotransmission of glutamate, prevented depression-like behavior in a rat model (Banasr et al., 2010). The glial hypothesis, mainly pioneered by researchers such as Sanacora and Raijkowska (Rajkowska et al., 1999; Kugaya and Sanacora, 2005; Rajkowska and Miguel-Hidalgo, 2007), thus serves as a crucial addition to other neuronal concepts of primarily serotonergic dysfunction. As proposed by some authors the complex situation in both neuronal and glial compartments, which forms the basis for task-related responses, can be investigated at rest (Raichle and Mintun, 2006; Raichle and Snyder, 2007; Shulman et al., 2007). The concept of spontaneous resting-state fluctuations first described by Biswal et al. (1995) seems ideal to investigate alterations of this system on the functional level (Fox and Raichle, 2007).

Resting-state functional connectivity (rsFC) is defined as the correlation of spontaneous low frequency BOLD signal time courses of distinct brain regions. In major depression, altered resting-state behavior in the default mode components has been shown (Greicius et al., 2007). Indications for a cortico-limbic dysfunction (Mayberg, 1997) were also found during task and rest (Anand et al., 2005a,b). These findings are related to a large number of altered functional responses in emotional core regions including medial prefrontal cortex and limbic structures (Rigucci et al., 2009), but most of these remain outside the classic task-positive attention regions such as dorsal ACC, anterior insula (AI) or lateral prefrontal cortex. Tasknegative regions respond with a decrease in BOLD signal to attentional tasks while task-positive regions are specifically activated at the same time. While the exact nature of functional anticorrelations at rest and the effects of preprocessing strategies remain unclear to date (Fox et al., 2009; Murphy et al., 2009), the functional separation of task-positive and task-negative regions using task and restingstate studies (Fox et al., 2005; Buckner and Vincent, 2007; Uddin et al., 2009; Neumann et al., 2010) is well accepted. Disease severity related alterations of rsFC in this intrinsic organization of the brain were recently described for major depression (Zhou et al., 2010).

Complex impairments of emotional and self awareness underlie the clinical symptoms of anhedonia, rigid affect or ruminations as well as negative self concept and altered social interactions. Taking this into account it is highly important to investigate the relationship of pgACC pathologies to dysfunctions in other domains especially those involved in the processing of emotions. Given the increasing evidence for the involvement of the insular cortex in the conscious processing of affect as well as the generation of percepts of self awareness in a subjective timeframe (Craig, 2009), one has to hypothesize functional abnormalities in the AI. In a recent study, Grimm et al. (2009a,b) showed altered neural responses in the salience network (Seeley et al., 2007) that are related to impaired judgments of self-relatedness of emotional stimuli. This study further provided evidence for left lateralized effects, which are well in accordance with a forebrain asymmetry of emotional valence and parasympathetic processing in left AI, which is also the putative target of vagal nerve stimulation in MDD (Craig, 2005).

The majority of depressive symptoms are present even in the absence of specific tasks when the patients are mainly engaged in exaggerated, negatively colored self-referential processing, continuous negative moods, and ruminative thoughts. Therefore it seems important to investigate the putative neural substrates during such abnormal functioning, i.e. at rest. Such baseline alterations, characterized by impaired rsFC between pgACC and AI, might be directly related to abnormal metabolite or cellular measures.

We thus aimed to investigate the relationship of altered baseline processes displayed in rs $\mathrm{fMRI}$ with the metabolic representations of neuronal-glial functioning seen in MRS. Pregenual ACC is a primary region of interest due to the vast literature on functional and metabolite abnormalities. It was thus tested if abnormal glutamatergic levels in pgACC would influence rsFC with $\mathrm{AI} /$ frontal operculum. The second region, as part of the task-positive, attention set network, was chosen because of strong direct anatomical connections with ACC in the macaque and human brain (Mesulam and Mufson, 1982; Mufson and Mesulam, 1982; Augustine, 1996) as well as its proposed role for the switching between anticorrelated networks (Sridharan et al., 2008).

Given the hypothesized primary deficit in the pgACC, we intend to further test if the putative abnormal anticorrelative behavior could be traced back to a molecular deficit in pgACC without direct relations to the metabolite profiles in AI. The aim was to test the specificity of glutamatergic deficiencies as symptoms of glial-neuronal pathology in pgACC. This would support the concept of altered functional network behavior outside the pgACC, at least in the salience network, as a consequence of a primary neuronal glial decline in pgACC in the case of MDD and may help to identify disease specific abnormalities in otherwise overlapping findings of abnormal resting-state behavior.

To prove the clinical relevance of such functional and metabolic abnormalities, we further tested their relation to markers of clinical severity of depression. During treatment response or remission, as indicated by decreasing scores of the HAMD, many of the 
initially described affective symptoms disappear whereas a number of cognitive-attentional deficits persist. Because of this observation we hypothesized a primary glutamatergic deficit in an affective region, i.e., pgACC, which is directly related to clinical severity and which drives the extent of abnormal rsFC between pgACC and AI. We further hypothesize that Glx concentrations in AI, which has stronger connections to other cognitive regions, are not related to severity or rsFC. The primary focus on pgACC is supported by the fast and strong effects of the glutamate modulating NMDA antagonist ketamine on HAMD scores in treatment resistant MDD patients (Zarate et al., 2006) since ketamine leads to increases of Glu concentrations in exactly this region (Rowland et al., 2005).

\section{MATERIALS AND METHODS SUBJECTS}

Twenty-two subjects with an acute MDD episode were recruited from the inpatient and outpatient department of psychiatry at the University of Magdeburg. Primarily, these patients were clinically diagnosed according to the ICD-10 criteria (WHO, 1992). Exclusion criteria were major medical illness, history of seizures, medication with glutamate modulating drugs (ketamine, riluzole, etc.) or benzodiazepines prior electroconvulsive therapy (ECT) treatments and pregnancy, as well as all contraindications against MRI. Specific psychiatric exclusion criteria consisted of atypical forms of depression, any additional psychiatric disorder, and a history of substance abuse or dependence. For a better specification of the depressive symptoms, all patients were rated by clinical questionnaires, among them the Hamilton depression scale. The HAMD was first developed by Hamilton (1960) to rate the acute state of depressive patients. Since then it has been known to accurately describe the disease severity of patients with an acute depressive episode. Moreover, the scores reflect the effects of druginduced improvement in disease. We used the German version of the 21-item HAMD (CIPS, 1996) for clinical subgroup analysis.

Twenty-two healthy subjects without any psychiatric, neurological, or medical illness were self-referred from study advertisements. All volunteers completed the mini-international neuropsychiatric interview (MINI) to specifically ensure the absence of any ICD-10 psychiatric disorders (Sheehan et al., 1998). The study was approved by the institutional review board of the University of Magdeburg and all subjects gave written informed consent before inclusion. All subjects underwent fMRI and MRS in an identical paradigm. All patients were medicated using SSRI, NRI, and SNRI alone or with new generation antidepressants including agomelatine or lithium. Due to specific MRS quality criteria (see below) 19 patients were finally included to the analysis while for one subject rs fMRI data could not be used. The composition of the sex- and age-matched groups is described in Table 1. Neither the controls compared to all patients ( $t$-test, $P=0.137$ ) nor the subgroups of healthy controls, mildly and severely depressed patients (one-way ANOVA, $F=1.49$, $P=0.238$ ) differed significantly by age.

\section{RESTING-STATE FMRI DATA ACQUISITION AND ANALYSIS}

Themeasurementswere performed on a 3 TeslaSiemens MAGNETOM Trio scanner (Siemens, Erlangen, Germany) with an eight-channel phased-array head coil. For acquisition of the resting-state fMRI data, the subjects were told to lie still in the scanner with their eyes
Table 1| Demographic and clinical description of subjects meeting the quality criteria

\begin{tabular}{lllll}
\hline & $\begin{array}{l}\text { Healthy } \\
\text { controls }\end{array}$ & $\begin{array}{l}\text { Patients } \\
\text { HAMD } \leq \mathbf{1 5}\end{array}$ & $\begin{array}{l}\text { Patients } \\
\text { HAMD }>\mathbf{1 5}\end{array}$ & All patients \\
\hline Number & 22 & 8 & 10 & 18 \\
Male/Female & $12 / 10$ & $5 / 3$ & $5 / 5$ & $10 / 8$ \\
Mean age & 34.14 & 37.62 & 40.5 & 39.22 \\
{$[S D]$} & {$[6.67]$} & {$[12.82]$} & {$[13.1]$} & {$[12.67]$} \\
Mean HAMD & 0 & 12.75 & 20.7 & 17.17 \\
{$[S D]$} & & {$[1.83]$} & {$[2.95]$} & {$[4.74]$} \\
\hline
\end{tabular}

closed. Functional time series of 488 time points were acquired with an echo-planar imaging sequence. The following acquisition parameters were used: echo time $=25 \mathrm{~ms}$, field of view $=22 \mathrm{~cm}$, acquisition matrix $=44 \times 44$, isometric voxel size $=5 \times 5 \times 5 \mathrm{~mm}^{3}$. Twenty-six contiguous axial slices covered the entire brain with a repetition time of $1250 \mathrm{~ms}$ (flip angle $=70^{\circ}$ ). The first five acquisitions were discarded to reach steady state and limit T1 effects. High resolution T1-weighted structural MRI scans of the brain were acquired for structural reference using a 3D-MPRAGE sequence $(\mathrm{TE}=4.77 \mathrm{~ms}$, TR $=2500 \mathrm{~ms}$, $\mathrm{T} 1=1100 \mathrm{~ms}$, flip angle $=7^{\circ}$, bandwidth $=140 \mathrm{~Hz} /$ pixel, acquisition matrix $=256 \times 256 \times 192$, isometric voxel size $\left.=1.0 \mathrm{~mm}^{3}\right)$.

Data was analyzed using the data processing assistant for resting-state fMRI (DPARSF ${ }^{1}$, Yan Chao-Gan, State Key Laboratory of Cognitive Neuroscience and Learning, Beijing Normal University, China) which includes resting-state fMRI data analysis toolkit (REST, by Song Xiao-Wei et al. ${ }^{1}$ ). Both toolboxes work on basis of the spm 5 software package (Wellcome Trust Center for Neuroimaging, London, England). Functional data was corrected for differences in slice acquisition time, motion-corrected using a least squares approach and a six-parameter (rigid body) linear transformation, spatially normalized and smoothed by using a 4-mm full-widthat-half-maximum Gaussian kernel. The data was linear detrended and filtered by a band pass filter $(0.01-0.08 \mathrm{~Hz})$ to eliminate low frequency fluctuations. The preprocessing steps followed the standard protocol published by Yan and Zang (2010). Accordingly, an additional regression of nuisance covariates was applied during which the functional data was corrected for the six head movement parameters and for global mean signal as well as for white matter and cerebrospinal fluid signal. This correction is discussed to elicit negative correlations between the time courses of normally anticorrelated resting-state network regions (Murphy et al., 2009).

By merging the individually placed spectroscopy voxel positions in normalized space, two regions of interest (ROI) where defined. The pgACC which belongs to the medial prefrontal cortex is localized in the rostral part of Brodmann area 24 and was defined by the Montreal Neurological Institute (MNI) coordinates $(x, y, z): 0,41$, 9. The ROI for left AI was placed with the center at 39, 19, 4. Both ROIs had a radius of $10 \mathrm{~mm}$ resulting in a volume of approximately $4 \mathrm{ml}$, which is in line with the size of the MRS voxels.

To compute the rsFC of the ROIs, the time course of every ROI was extracted and a correlation analysis was run to elicit the correlation coefficient of the different time courses. The correlation coefficient

${ }^{1}$ www.restfmri.net 
therefore reflects a statistical interrelation of the BOLD time courses in the specific ROIs but no direction or causality. Correlation coefficients close to 1 hereby mean that the time courses have a high synchronicity. Brain morphology was assessed using the fully automated Civet pipeline of the Montreal Neurological Institute (Lerch and Evans, 2005; Ad-Dab'bagh et al., 2006). Following this, the tissue composition and cortical thickness of the whole brain and the specified MRS voxels could be included in the analysis as cofactors.

\section{MRS DATA ACOUISITION AND ANALYSIS}

Single voxel proton MRS data was acquired at rest for each subject from a first volume of interest of $10 \times 20 \times 20 \mathrm{~mm}^{3}=4.0 \mathrm{ml}$ which was placed in bilateral pgACC and from a second one of $15 \times 10 \times 20 \mathrm{~mm}^{3}=3.0 \mathrm{ml}$ which was placed in the frontal operculum of the left AI (Figure 1). The location and extent of the pgACC voxel was oriented on our previous findings of altered self awareness, impaired BOLD responses and altered glutamatergic levels in depressed patients (Grimm et al., 2009a,b; Walter et al., 2009).

The measurements were performed on a $3 \mathrm{~T}$ Siemens MAGNETOM Trio scanner. A PRESS (point-resolved spectroscopy) sequence with the following acquisition parameters was used for both voxels: echo time $=80 \mathrm{~ms}$, repetition time $=2000 \mathrm{~ms}$, 256 averages, bandwidth $=1200 \mathrm{~Hz}$, acquisition time $=853 \mathrm{~ms}$ and water suppression. The echo time of $80 \mathrm{~ms}$ was used according to the results of Schubert et al. (2004). Manual shimming was performed to improve magnetic field homogeneity set by the automatic shim routine. Additionally, water reference data with radiofrequency pulses for water suppression switched off ( $\mathrm{TR}=10 \mathrm{~s}$, four averages) were acquired for eddy current correction. The acquisition time for every voxel added up to $8 \mathrm{~min}$ and $40 \mathrm{~s}$. Spectra were analyzed using LCModel version 6.1.0 (Provencher, 1993). Sixteen different metabolites (Creatine, Glutamate, myoInositole, Lactate, NAA, Phosphocholine, Taurine, Aspartate, GABA, Glutamine, Glucose, Alanine, NAAG, Phosphocreatine, Guanine, and Glycerophosphocholine) were fitted using a basis set including all these substances. Since creatine has been described to be an appropriate internal reference for the measured metabolite concentrations in MDD (Yildiz-Yesiloglu and Ankerst, 2006), all metabolite values are given as creatine $(\mathrm{Cr})$ ratios. Cramer-Rao lower bounds (Cavassila et al., 2001), an estimate of the fitting error, was used as a quality criterion to exclude datasets with unreliable quantification results. Hence, analyses of group effects were restricted to subjects meeting the strict quality criteria to indicate reliable spectral identification (Cramer-Rao lower bounds $<20 \%$ ). We did not have to reject spectra due to a poor line width since all used spectra had a full-width-at-half maximum smaller than $12 \mathrm{~Hz}$. The signal to noise ratio was larger than eight for all subjects.

\section{STATISTICAL ANALYSIS}

Correlation coefficients of BOLD time courses of pgACC and AI, metabolite levels in both of the regions and HAMD scores were correlated by applying Pearson correlation analysis (SPSS, version 15; SPSS Inc, Chicago, IL, USA). Statistical significance was set at $P<0.05$. Significant correlations were controlled for confounding effects of age by partial correlation. Extreme values were defined as exceeding the 25th or 27th percentile by more than three interquartile ranges in either direction and were consequently removed. All correlations were calculated separately for healthy controls and MDD patients. To test for intergroup differences, one-way ANOVA was first run followed by twosample $t$-tests. The significance threshold was also set at $P=0.05$.

\section{RESULTS \\ PREDICTION OF RESTING-STATE CONNECTIVITY BETWEEN pgACC AND ANTERIOR INSULA IN DEPRESSION BY MRS}

The Glx to creatine ratio in pgACC predicted the functional connectivity between this region and the left AI (Figure 2). This relationship between metabolite profiles and functional connectivity was specific for depressed patients $(r=-0.51, P=0.031)$ while healthy controls did not show this effect $(r=-0.15, P=0.49)$. The same effect could also be observed when only Glu ratios were looked at for the depressed patients $(r=-0.488, P=0.04)$ and remained insignificant for the healthy controls $(r=-0.111, P=0.62)$.

Lower Glx ratios in pgACC predicted weaker negative correlations, i.e., functional connectivity between pgACC and AI. Patients with the lowest Glx ratios even showed positive correlation coefficients. In contrast, Glx measures in the insula failed to predict the connectivity between the two regions $(P=0.7)$. No correlations were found between gray matter contents of the MRS single voxels and functional connectivities or Glx ratios. Also, patients and controls did not differ in their relative gray and white matter tissue compositions in the two voxels.

Since age has been described to be a factor influencing functional connectivity (Damoiseaux et al., 2008) its effects were explored specifically, but no correlation of age with connectivity values or Glx ratios was found in our samples of either patients or controls.

\section{ALTERED CONNECTIVITIES AND RELATED METABOLITE LEVELS WITH RELEVANCE FOR CLINICAL SEVERITY OF DEPRESSION}

Supporting the clinical relevance of the predescribed findings, a significant correlation between HAMD and rsFCs between AI and pgACC could be detected ( $r=-0.617, P=0.006)$ (Figure 3A).

Furthermore symptom severity was predicted by relative Glu and Glx levels only in pgACC (Glu: $r=-0.51, P=0.031 /$ Glx: $r=-0.44$, $P=0.068$ ) (Figure 3B) while no such effect was seen for Glx or Glu values in the AI (Glu: $r=0.092, P=0.72 / \mathrm{Glx}: r=0.052, P=0.84$ ). To test the relevance of altered connectivities and related metabolite levels for clinical severity of depression directly, we divided the patients into a severely depressed and a mildly depressed group with a median split according to HAMD scores (median: 16). This resulted in two groups (eight and 10 patients) with no significant differences in age, size, or gender composition (Table 1).

As illustrated in Figures 4 and 5, one-way ANOVAs revealed group effects for Glx and rsFC with altered Glu and Glx ratios in $\operatorname{pgACC}$ (Glu: $F=8.35, P=0.001 / \mathrm{Glx}: F=6.993, P=0.003$ ). Different FCs between both regions $(F=4.64, P=0.016)$ were detected for severely depressed patients compared to both other groups, while no significant effects were found for Glu or Glx ratios in AI (Glu: $F=0.8, P=0.457 / G l x: F=0.91, P=0.41$ ).

\section{ALTERED FUNCTIONAL CONNECTIVITY BETWEEN PgACC AND AI IN SEVERELY DEPRESSED PATIENTS}

A direct comparison of the functional connectivities of pgACC and AI revealed significantly higher correlations of signal time courses for severely depressed patients with HAMD scores 16 or 

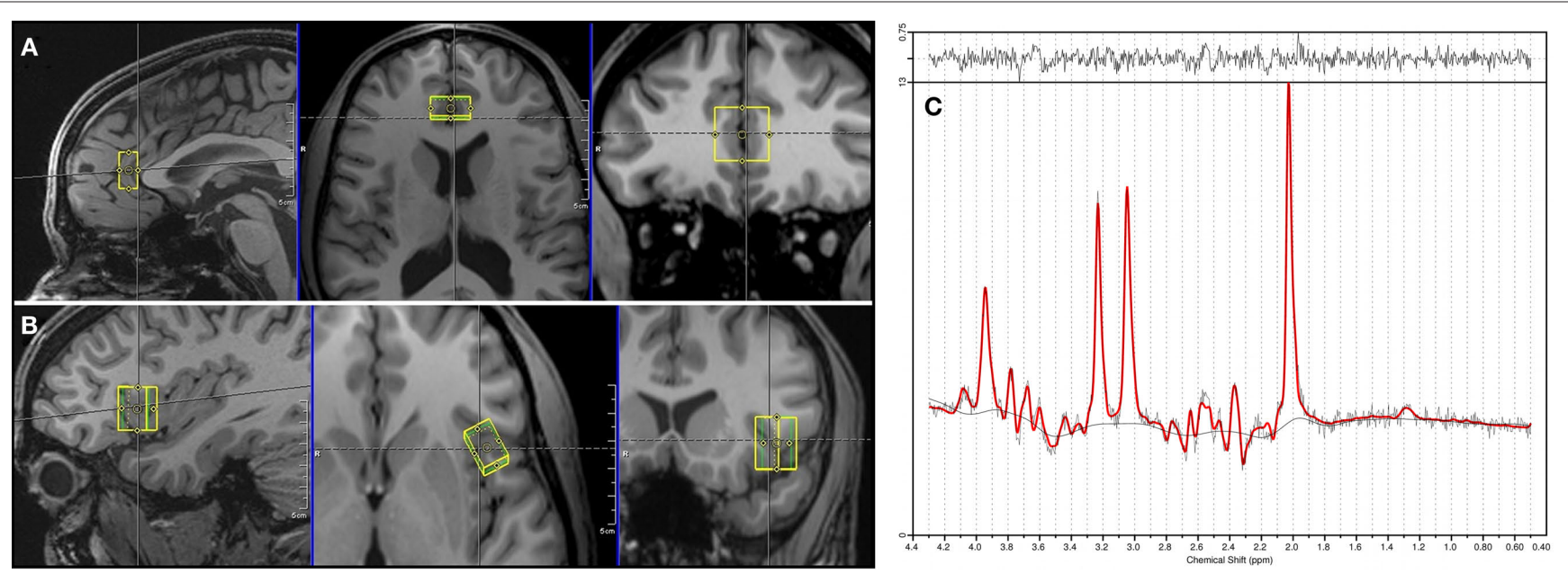

FIGURE 1 | Positioning of the spectroscopy voxels (A: pgACC, B: Al) and a representative spectrum of pgACC (C).
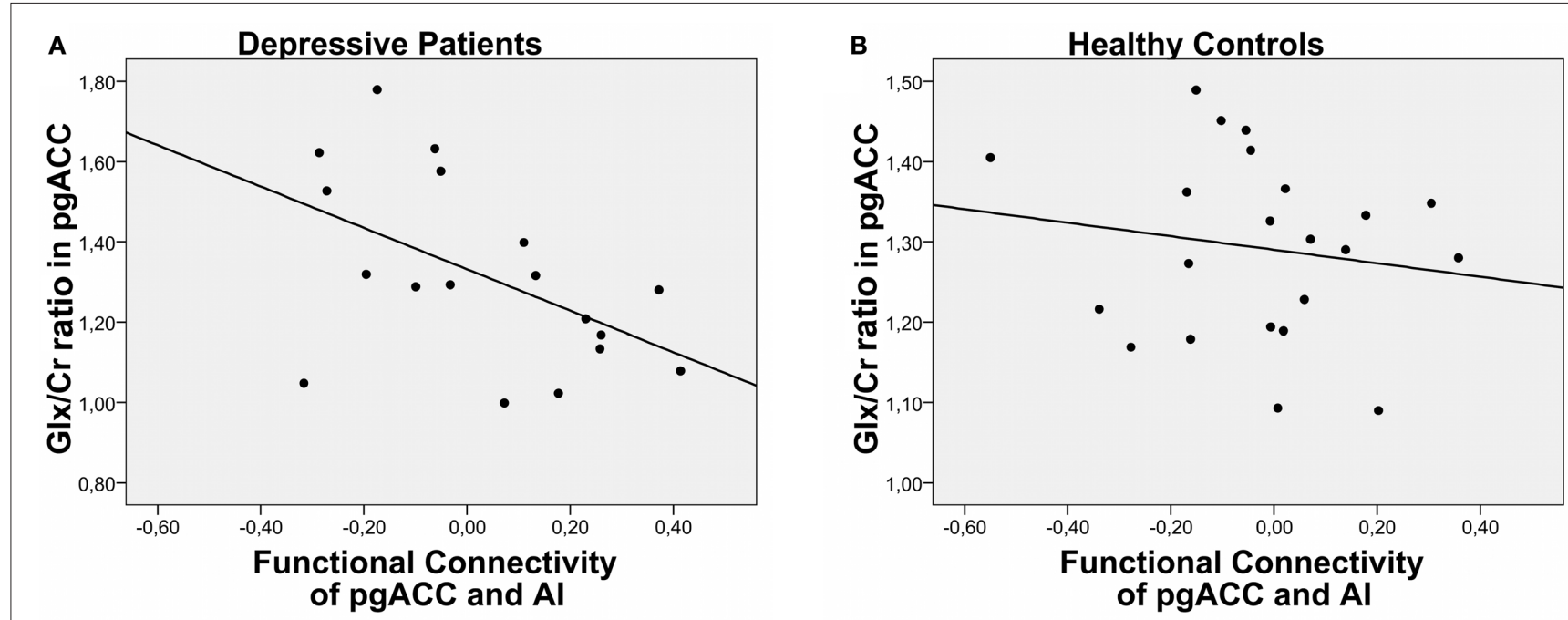

FIGURE 2 | Correlation of functional connectivity between pgACC and Al and GIx/Cr ratios for depressed patients (A) and healthy controls (B) ratios was found in our samples of either patients or controls.

higher $($ mean FC $[\mathrm{SD}]=0.15[0.21])$ than for healthy controls $($ mean $\mathrm{FC}=-0.03[0.21])(T=-2.26, P=0.03)$ and mildly depressed patients (mean FC $=-0.12[0.14])(T=-3.1, P=0.007)$ (Figure 4). No significant differences in FCs were found between the mildly depressed group and healthy controls.

\section{DEPRESSION SEVERITY IS RELATED TO THE METABOLITE PROFILE IN pgACC}

Similar to the functional connectivities, a direct comparison of the Glu and Glx levels in pgACC revealed lower ratios for acutely depressed patients with HAMD scores 16 or higher than for healthy controls (Glu: $T=2.59, P=0.015 / \mathrm{Glx}: T=1.75$, $P=0.09)$ as well when compared to mildly depressed patients (Glu: $T=3.73, P=0.002 / \mathrm{Glx}: T=2.941, P=0.01$ ). In contrast to the connectivity findings a significant difference of Glu and Glx levels between healthy controls and mildly depressed patients was observed (Glu: $T=-2.42, P=0.022 / G l x: T=-2.454$, $P=0.037)$.

Although we did not have a priori hypotheses for abnormal values of myo-Inositole, creatine or NAA, we also tested for differences in these metabolites in one-way ANOVA's $(P>0.22)$. We could however not find any significant alterations for the patients (see also Table 2).

\section{DISCUSSION}

Our findings provide first evidence for abnormal glutamatergic modulation of resting-state brain activity in depression. In severely depressed patients, functional connectivity between pgACC and AI was linearly correlated with $\mathrm{Glx} / \mathrm{Cr}$ ratios in the pgACC. Such a correlation was not evident in healthy controls. When exploring the clinical relevance of 


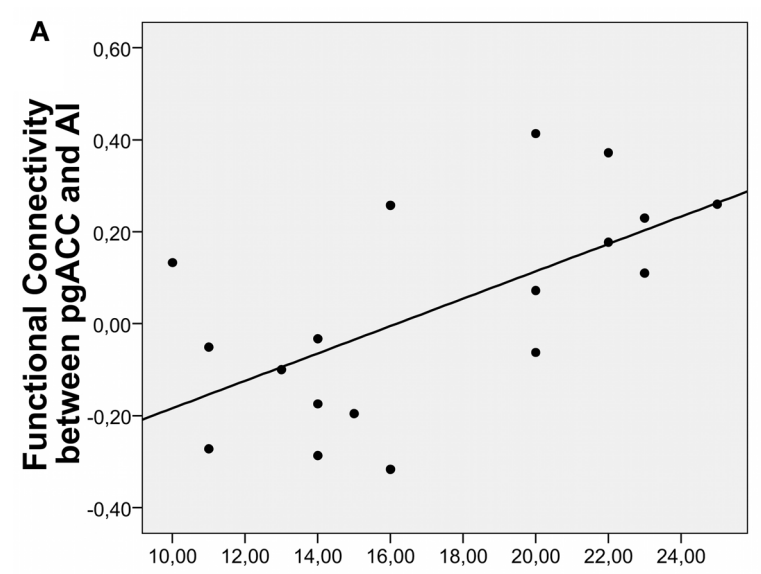

Hamilton Depression Scale Score

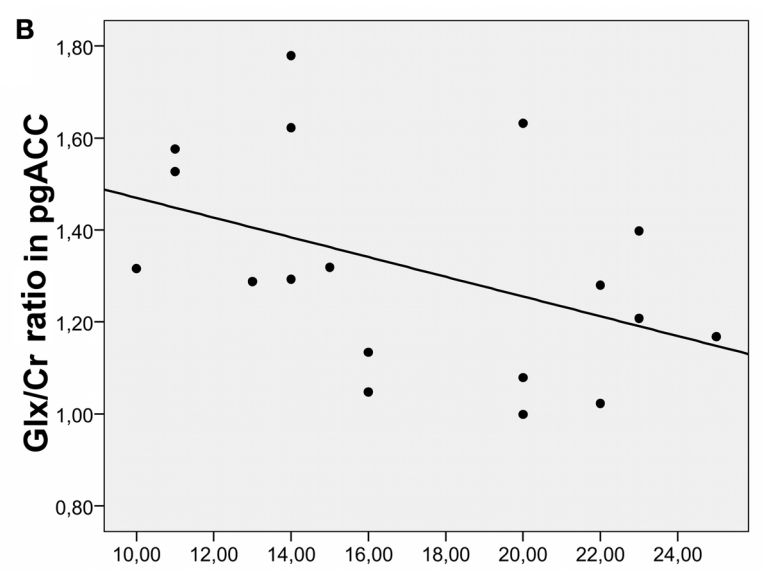

Hamilton Depression Scale Score

FIGURE 3 | Correlation of clinical severity of depression with RSFC (A) as well as with Glx/Cr ratios in pgACC (B).

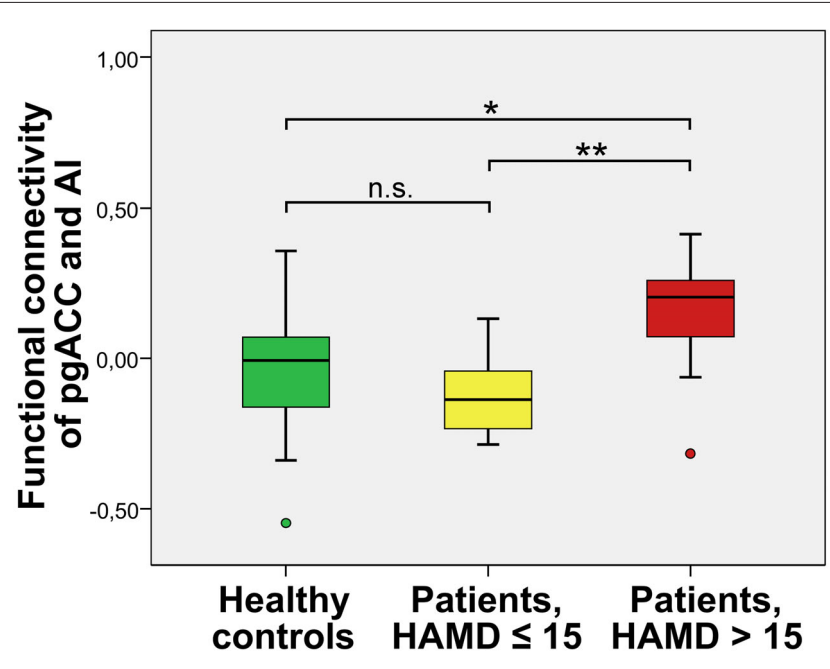

FIGURE 4 | Severely depressed patients with altered functional connectivity between pgACC and Al (box plot showing median, interquartile range, sample minimum and maximum; ${ }^{*} P<0.05$, ${ }^{*} P<0.01$, n.s.: not significant.

this metabolic impact on resting-state connectivity, a trend for linear correlation of HAMD with both factors was found. Consequently, direct comparison of $\mathrm{Glx} / \mathrm{Cr}$ ratios revealed lower concentrations in severely depressed patients as compared to healthy controls and mildly depressed patients. However, this glutamatergic deficit was only present in pgACC and was accompanied by abnormal functional connectivities at rest. Only severely depressed patients showed weaker negative or even positive correlations of time courses in pgACC and AI.

The evidence of a glutamatergic deficit and its importance for functional alterations in ACC reported here is in line with similar findings from our group in an unmedicated highly depressed patient population (Walter et al., 2009). In this group of patients with HAMD scores of 24 or higher, we found that lower levels of glutamine and glutamate predicted weaker functional responses in

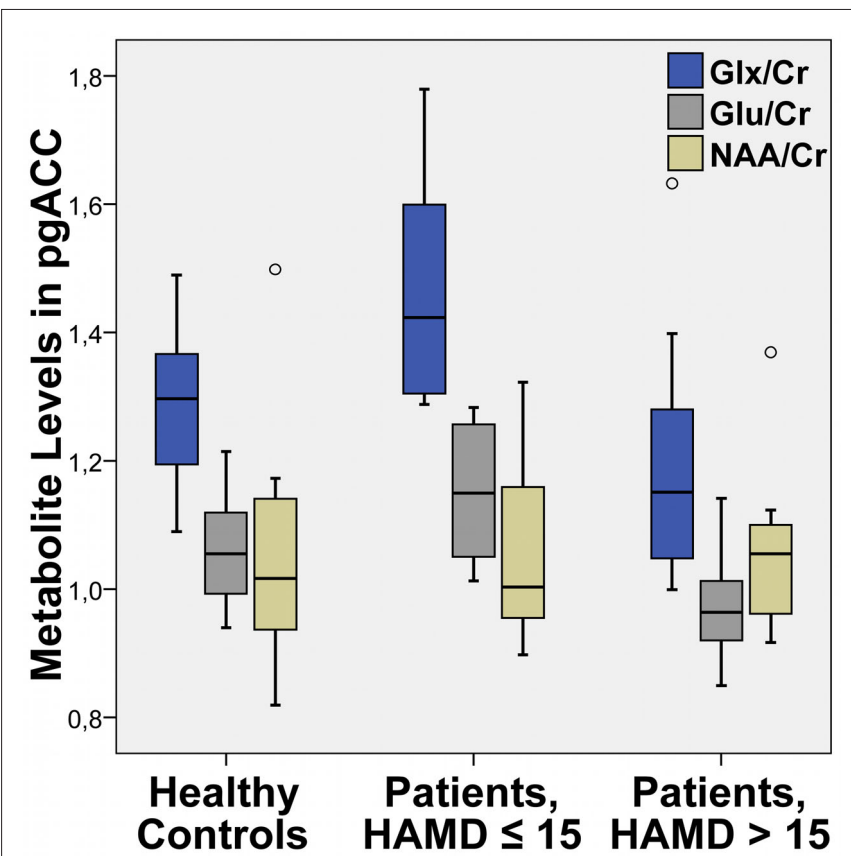

FIGURE 5 | Mean levels of the sum value of Glu and Gln (GIx), glutamate (Glu) and NAA relative to creatine (Cr) in pgACC (box plot showing median, interquartile range, sample minimum and maximum).

the pgACC using a 2D JPRESS MRS sequence. Functional responses were weaker in patients compared to healthy controls. In support of an effect of anhedonia on brain activity, specifically highly anhedonic patients showed impaired negative BOLD responses during a cognitive task. This is mirrored by our findings in this new study that showed that even the more general marker of HAMD scores was able to turn out subgroups differing in their baseline connectivity and metabolite levels. Correlation of rsFC and $\mathrm{Glx} / \mathrm{Cr}$ ratios was specific to the patient group and pathologically altered rsFC were found to be prominent only in the severely depressed group. 
Table 2 | Mean levels of fitted metabolites with valid spectra (CRLB $<20$ in at least $75 \%$ of the subjects).

\begin{tabular}{|c|c|c|c|c|c|c|}
\hline & \multicolumn{2}{|c|}{ Healthy controls } & \multicolumn{2}{|c|}{ Patients HAMD $\leq 15$} & \multicolumn{2}{|c|}{ Patients HAMD > 15} \\
\hline & pgACC & Al & pgACC & Al & pgACC & Al \\
\hline Glutamate/Cr & $1.06 \pm 0.08$ & $0.82 \pm 0.61$ & $1.15 \pm 0.11$ & $1.01 \pm 0.45$ & $0.98 \pm 0.09$ & $1.06 \pm 0.19$ \\
\hline myo-Inositole/Cr & $0.71 \pm 0.14$ & $0.49 \pm 0.34$ & $0.71 \pm 0.07$ & $0.58 \pm 0.31$ & $0.67 \pm 0.09$ & $0.70 \pm 0.15$ \\
\hline $\mathrm{NAA} / \mathrm{Cr}$ & $1.04 \pm 0.15$ & $0.85 \pm 0.59$ & $1.06 \pm 0.15$ & $1.03 \pm 0.44$ & $1.06 \pm 0.13$ & $1.11 \pm 0.14$ \\
\hline
\end{tabular}

This leads to at least three main conclusions:

Firstly, we extend the observations of impaired brain function of pgACC in a resting-state condition: In contrast to the abnormalities within DMN or cortico-limbic connectivities which were previously reported (Greicius et al., 2007; Anand et al., 2009), our study shows altered correlations between pgACC and AI. These regions belong to different networks with opposing behavior upon external stimulation. While pgACC has strongest rsFC with the ventromedial prefrontal part of the default mode network (Margulies et al., 2007) the AI is positively correlated to the task-positive attention set network. The AI belongs to this network together with the dorsal ACC (Dosenbach et al., 2008) and both are of specific importance for the processing of a stimulus' salience (Seeley et al., 2007). A newly proposed network model of insula function describes its role in saliency and switching between networks (Menon and Uddin, 2010). This is in line with the observation that depressive patients are less able to down-regulate the DMN activity during tasks (Sheline et al., 2009). Our results may thus help to explain specific MDD symptoms like ruminating thoughts and the clinical impression of depressed patients being "caught" in their depressed mood. Such hyper stable assignment of the AI to the default mode network may impair its flexibility in coordinating the patients' attention being directed either towards the external world or the internal perception of self-related processes.

Secondly, we extend the previously described glutamatergic modulation of abnormal fMRI responses (Walter et al., 2009) to a baseline condition. This supports the hypothesis that altered basic conditions of the network architecture lead to abnormal functional responses, such as, for example, decreased negative BOLD responses during cognitive tasks in a region belonging to the DMN (Mennes et al., 2010). Resting-state alterations and impaired reactivity to external stimulations in the pgACC were thus both shown to depend on glutamatergic metabolite levels. Therefore we assume that rsFC of this region is able to directly predict the degree of excitability in terms of subjective reports and functional responses in patients during stimulation (Hampson et al., 2006). This direct prediction still remains to be shown for MDD and related symptoms in the future.

Thirdly, we substantiate the importance of altered metabolite and functional levels in this key region of the ACC to the severity of depressive symptoms, since in both $\mathrm{rsFC}$ and $\mathrm{Glx} / \mathrm{Cr}$ ratios the severely depressed patients (HAMD scores of 16 or higher) showed the strongest alterations. In an animal model glial changes that where induced by chronic and unpredictable stress could be reversed or blocked by treating the rats with riluzole which influences the glutamatergic neurotransmission (Banasr et al., 2010). In terms of clinical treatment this pregenual glial-neuronal deficit would favor the use of glutamatergic drugs in patients showing such functional and neurotransmitter patterns. A previous study showed that a relative hypometabolism in exactly this region discerns treatment resistant patients from those responsive to selective serotonin reuptake inhibitor (SSRI) treatment (Mayberg, 1997). Our findings can further explain the specific efficacy of glutamatergic drugs such as ketamine in severe, treatment resistant depression (Zarate et al., 2006) which leads to increased glutamate/glutamine levels in pgACC (Rowland et al., 2005).

The depicted molecular deficit in the pgACC is related to functional connectivity between this region and AI. Interestingly, we did not find an interdependence of FCs and AI ratios of Glx or Glu to $\mathrm{Cr}$. This is accompanied by prominent histoarchitectonic findings in the pgACC from a large number of postmortem studies. These suggest reduced glial densities and expression of enzymes allowing for glutamate reuptake and conversion to glutamine (Rajkowska et al., 1999; Cotter et al., 2001; Rajkowska and Miguel-Hidalgo, 2007). In this context, the reversible reductions of Glx would mirror a hypometabolic state with lowered glutamine cycling between glial cells and neurons, which by itself is highly energy demanding (Raichle and Mintun, 2006). Such altered baseline metabolism would thus result in altered functional connectivities, given that these can be regarded elementary for normal adaptive brain functions in healthy human beings. It has recently been reported that inter-individual differences in resting-state functional connectivity predict task-induced BOLD activity (Mennes et al., 2010). While reduced anticorrelations could be regarded as signs of decreased flexibility of the brain to react upon external stimulations, these altered connectivities could additionally be considered as the underlying sources of reduced functional responses upon explicit stimulation and consequently altered affective processing in MDD. Interestingly, we did not observe significant differences in NAA, myo-Inositole or creatine levels suggesting a specific relationship of glutamatergic mechanisms and abnormal rsFCs in depression. Stable concentrations of creatine in all groups also validate the approach of reporting the metabolite concentrations relative to creatine, since, in principle, the altered ratios could be a result of abnormal levels of creatine due to its involvement in brain metabolism.

In schizophrenia, similar mechanisms of altered connectivity between fronto-parietal and default mode cortical networks have been observed. In an independent component analysis, healthy subjects showed less correlation between these two networks than 
patients when correlations are generally positive (Jafri et al., 2008). At the same time they showed more negative values of connectivity than schizophrenic patients when global signal removal has caused the correlation values to be negative (Whitfield-Gabrieli et al., 2009). Here, we show such alterations in pgACC and AI and further provide a molecular dimension focusing glutamatergic mechanisms that are in line with existing postmortem and pharmacological evidence.

Notably, the anticorrelation between pgACC and AI is not a robust finding in the literature and also in our current sample, only a subset of healthy controls showed anticorrelations after global mean regression. Some regions belonging to the DMN like medial prefrontal and posterior cingulate cortex show consistently strong anticorrelations with task-positive regions like dorsal ACC and dorsolateral prefrontal cortex (DLPFC). In contrast to such regions, pgACC is variously correlated with the opposed networks. It shows less direct anticorrelations, but nevertheless strong anatomical connections and closer anatomical vicinity to a large set of cortical regions. Similarly, AI is functionally connected to dorsal ACC, and to a weaker extent, to DLPFC. Taking their anatomical interposition between task-positive and task-negative networks into account, this may support pgACC and AI's pivotal role in communicating the anticorrelated behavior (Menon and Uddin, 2010; Sridharan et al., 2008) of their associated networks. Such perspective might be in support of interpreting our results as representation of disturbed network modulation in MDD. The role of global mean regression in increasing observation of so called anticorrelations is a subject of current investigation. While there is evidence that these may not be present without the regression of global means (Murphy et al., 2009), others argue for an existence of these negative correlations in the absences of global mean regression if other means of physiological noise correction are considered (Chang and Glover, 2009; Fox et al., 2009). It is also possible to take a more conservative perspective and to accept that any additional introduction of nuisance covariates may lead to a reduction in correlation values and further towards negative values (Weissenbacher et al., 2009). The observed interdependence of rsFCs and Glx values may then at best be interpreted by the different degrees of assignment of the AI to task positive or default mode networks.

The role of the AI cortex in the pathophysiology of depression is less evident in the previous literature. One study reported relative hypermetabolism of AI in MDD which is correlated with disease severity (Brody et al., 2001). The absence of extensive postmortem findings in the AI might also indicate that the altered rsFC between pgACC and AI is driven by pgACC dysfunction. This may explain why clinical symptoms found in major depression exceed core functions of the pgACC, such as attribution of hedonic valences. Impaired AI-pgACC connectivity during emotional stimulation, internally or externally generated, may thus be responsible for reduced intensities or color of experienced emotions, since the AI may be crucial for the conscious experience of emotions (Uddin and Menon, 2009).

Another proposed role of AI is the switching between DMN and attentional networks (Sridharan et al., 2008). Therefore the postulated impairment of anticorrelated resting-state behavior could also be relevant in a number of cognitive tasks. Specific tasks require adaptive and efficient switching and transient reassignment of different network modules. Such cognitive and attentional deficits are frequently observed in severe depression. Since the AI is part of the cingulo-opercular attention set system (Dosenbach et al., 2008) as well as the salience network (Seeley et al., 2007), changes of its functional connectivity may be responsible for commonly observed negative bias in MDD (Bermpohl et al., 2009).

Salvadore et al. (2009) reported rostral ACC activity in an elaborate magneto encephalography (MEG) study to be predictive of subsequent treatment response to ketamine. Based on our findings, such predictions may in principle be possible using the more direct detection of altered baseline processing, which in the case of resting-state fMRI can be feasible within less than $10 \mathrm{~min}$ and without patients' active contribution to a specific task.

It has to be critically acknowledged that all patients in the current study have been on antidepressant pharmacotherapy. Although we have previously reported glutamatergic abnormalities in an unmedicated sample (Walter et al., 2009), potential confounds of medication cannot be fully ruled out. It may be of interest that glutamatergic abnormalities can be found in both medicated and unmedicated patients. But future studies, powered for adequate subgroup analyses, should focus on potential dose or treatment effects of serotonergic agents with potential downstream effects on glutamate levels.

A major limitation concerns the interpretation of MRS glutamate values. Although previous studies employed 1D PRESS MRS sequences at field strengths of 3 Tesla or even lower reporting both Glx and Glu (Auer et al., 2000), the separation of Glu and Gln should be regarded critically given limited line separation. By using an optimal echo time of $80 \mathrm{~ms}$ the contributions of Gln or GABA to the Glu signal should be minimal (Schubert et al., 2004; Mullins et al., 2008). Although findings for Glu and Glx strongly converged in our current analysis and Glx levels are strongly dominated by glutamate since it is the most abundant metabolite in comparison to GABA, Gln, or NAAG, one should be aware that Glu and Glx may concern slightly different effects. In a recent study using 2D JPRESS sequences, it could be shown that Gln predicts fMRI amplitudes in pgACC for both patients and controls, while Glu was correlated with fMRI responses only in the patient group (Walter et al., 2009). Another study (Brennan et al., 2010) reports subtle changes in the Gln/Glu ratio, which might be buried in the Glx estimates. This strongly motivates the use of sequences with sufficient separation of Glu and Gln. In our study the covariance of Glu and Gln values in the Fisher information matrix reaches a critical value of 0.3 . Therefore a more conservative interpretation should rely only on the sum values of Glu and Gln despite the optimum echo time and high measurement repetitions of 256 samples. The use of a $2 \mathrm{D}$ approach that would make use of $\mathrm{J}$ coupling information was however not possible given that coverage of multiple regions plus additional resting-state fMRI acquisition would not be possible due to heavily increased acquisition times.

Although the findings of the present study are in line with predictions from previous task fMRI-MRS studies, future investigations should directly link task responses, resting-state behavior and specific clinical symptoms for better inference. Longitudinal treatment studies are also of specific interest because of the observed impact of depression severity on the glutamatergic abnormalities. Although only present on a trend level, we found that rsFCs in the mildly depressed subgroup were lower than the levels observed in healthy controls. While this might be well in line with slightly increased Glx and Glu levels, any interpretation in the direction of overshooting correlates of treatment response should be carefully limited given the setup of our study, which did not allow for balanced longitudinal 
observations. The mildly depressed group should not be confused with partially recovered patients. Recent observations by Brennan et al. (2010) however, would suggest increased glutamine-glutamate cycling in the course of recovery which subsequently may lead to increases as observed in the mildly depressed subgroup.

Future studies will also have to integrate as many regions as possible into such a multimodal framework. Given the technical constraints of the MRS measurements, whole brain coverage is not practical. The current approach may benefit from its strong hypothesis driven character but multivoxel acquisitions as provided by chemical shift imaging (CSI) sequences may help to investigate regional specificity of our findings.

Our study provides a first promising step to combine evidence from elaborate activation studies and baseline investigations, which do not only concern the functional architecture at rest

\section{REFERENCES}

Ad-Dab'bagh, Y., Lyttelton, O., Muehlboeck, J.S., Lepage, C., Einarson, D., Mok, K., Ivanov, O., Vincent, R. D., Lerch, J., Fombonne, E., and Evans, A. C. (2006). "The CIVET imageprocessing environment: a fully automated comprehensive pipeline for anatomical neuroimaging research," in Proceedings of the 12th Annual Meeting of the Organization for Human Brain Mapping, ed. M. Corbetta (Elsevier, Florence).

Anand, A., Li, Y., Wang, Y., Lowe, M. J., and Dzemidzic, M. (2009). Resting state corticolimbic connectivity abnormalities in unmedicated bipolar disorder and unipolar depression. Psychiatry Res. 171, 189-198.

Anand, A., Li, Y., Wang, Y., Wu, J., Gao, S., Bukhari, L., Mathews, V. P., Kalnin, A., and Lowe, M. J. (2005a). Activity and connectivity of brain mood regulating circuit in depression: a functional magnetic resonance study. Biol. Psychiatry 57, 1079-1088.

Anand, A., Li, Y., Wang, Y., Wu, J., Gao, S., Bukhari, L., Mathews, V. P., Kalnin, A., and Lowe, M. J. (2005b). Antidepressant effect on connectivity of the mood-regulating circuit: an FMRI study.Neuropsychopharmacology 30, 1334-1344.

Auer, D. P., Pütz, B., Kraft, E., Lipinski, B., Schill, J., and Holsboer, F. (2000). Reduced glutamate in the anterior cingulate cortex in depression: an in vivo proton magnetic resonance spectroscopy study. Biol. Psychiatry 47, 305-313.

Augustine, J. R. (1996). Circuitry and functional aspects of the insular lobe in primates including humans. Brain Res. Brain Res. Rev. 22, 229-244.

Banasr, M., Chowdhury, G. M. I., Terwilliger, R., Newton, S. S., Duman, R. S., Behar, K. L., and Sanacora, G. (2010). Glial pathology in an animal model of depression: reversal of stress-induced cellular, meta- bolic and behavioral deficits by the glutamate-modulating drug riluzole. Mol. Psychiatry 15, 501-511.

Banasr, M., and Duman, R. S. (2008). Glial loss in the prefrontal cortex is sufficient to induce depressive-like behaviors. Biol. Psychiatry 64, 863-870.

Bermpohl, F., Walter, M., Sajonz, B., Lucke, C., Hagele, C., Sterzer, P., Adli, M., Heinz, A., and Northoff, G. (2009). Attentional modulation of emotional stimulus processing in patients with major depression-alterations in prefrontal cortical regions. Neurosci. Lett. 463, 108-113.

Biswal, B., Yetkin, F. Z., Haughton, V. M., and Hyde, J. S. (1995). Functional connectivity in the motor cortex of resting human brain using echo-planar MRI. Magn. Reson. Med. 34, 537-541.

Brennan, B. P., Hudson, J. I., Jensen, J. E., McCarthy, J., Roberts, J. L., Prescot, A. P., Cohen, B. M., Pope, H. G., Renshaw, P. F., and Ongür, D. (2010). Rapid enhancement of glutamatergic neurotransmission in bipolar depression following treatment with riluzole. Neuropsychopharmacology 35, 834-846.

Brody, A. L., Saxena, S., Mandelkern, M. A., Fairbanks, L. A., Ho, M. L., and Baxter, L. R. (2001). Brain metabolic changes associated with symptom factor improvement in major depressive disorder. Biol. Psychiatry 50, 171-178.

Buckner, R. L., and Vincent, J. L. (2007). Unrest at rest: default activity and spontaneous network correlations. Neuroimage 37, 1091-1096; discussion 1097-1099.

Cavassila, S., Deval, S., Huegen, C., van Ormondt, D., and Graveron-Demilly, D. (2001). Cramér-Rao bounds: an evaluation tool for quantitation. NMR Biomed. 14, 278-283.

Chang, C., and Glover, G. H. (2009). Effects of model-based physiological noise correction on default mode network anti-correlations and correlations. Neuroimage 47, 1448-1459.

but also its molecular and finally its cellular representations. Future studies are necessary not only to replicate these findings in a larger sample but also to relate the observed mechanisms to pharmacological responses and more specific clinical characterizations.

\section{DISCLOSURE}

The study was funded by SFB 779 .

\section{ACKNOWLEDGMENTS}

We are grateful for the important suggestions and discussions with Dr. Helen Mayberg. We thank Dr. Cameron Craddock and Thomas M. Malone for their skillful technical support and help during preparation of the revised manuscript.

Choudary, P. V., Molnar, M., Evans, S. J., Tomita,H.,Li,J.Z., Vawter,M.P., Myers, R. M., Bunney, W. E., Akil, H., Watson, S. J., and Jones, E. G. (2005). Altered cortical glutamatergic and GABAergic signal transmission with glial involvement in depression. Proc. Natl. Acad. Sci. U.S.A. 102, 15653-15658.

CIPS. (1996). Internationale Skalen für Psychiatrie. Göttingen: Beltz Test $\mathrm{GmbH}$.

Cotter, D., Mackay, D., Landau, S., Kerwin, R., and Everall, I. (2001). Reduced glial cell density and neuronal size in the anterior cingulate cortex in major depressive disorder. Arch. Gen. Psychiatry 58, 545-553.

Craig, A. D. B. (2005). Forebrain emotional asymmetry: a neuroanatomical basis? Trends Cogn. Sci. (Regul. Ed.) 9, 566-571.

Craig, A. D. B. (2009). How do you feelnow? The anterior insula and human awareness. Nat. Rev. Neurosci. 10, 59-70.

Damoiseaux, J.S., Beckmann, C.F.,Arigita, E. J.S., Barkhof, F., Scheltens, P., Stam, C. J., Smith, S. M., and Rombouts, S. A. R. B. (2008). Reduced resting-state brain activity in the "default network" in normal aging. Cereb. Cortex 18 1856-1864.

Dantzer, R., O'Connor, J. C., Freund, G. G., Johnson, R. W., and Kelley, K. W. (2008). From inflammation to sickness and depression: when the immune system subjugates the brain. Nat. Rev. Neurosci. 9, 46-56.

Dosenbach, N. U. F., Fair, D. A., Cohen, A. L., Schlaggar, B. L., and Petersen, S. E. (2008). A dual-networks architecture of top-down control. Trends Cogn. Sci. (Regul. Ed.) 12, 99-105.

Fox, M. D., and Raichle, M. E. (2007). Spontaneous fluctuations in brain activity observed with functional magnetic resonance imaging. Nat. Rev. Neurosci. 8, 700-711.

Fox, M. D., Snyder, A. Z., Vincent, J. L., Corbetta, M., van Essen, D. C., and
Raichle, M. E. (2005). The human brain is intrinsically organized into dynamic, anticorrelated functional networks. Proc. Natl. Acad. Sci. U.S.A. 102, 9673-9678.

Fox, M. D., Zhang, D., Snyder, A. Z., and Raichle, M.E. (2009). The global signal and observed anticorrelated resting state brain networks. J. Neurophysiol. 101, 3270-3283.

Greicius, M. D., Flores, B. H., Menon, V., Glover, G. H., Solvason, H. B., Kenna, H., Reiss, A. L., and Schatzberg, A. F. (2007). Resting-state functional connectivity in major depression: abnormally increased contributions from subgenual cingulate cortex and thalamus. Biol. Psychiatry 62, 429-437.

Grimm, S., Boesiger, P., Beck, J., Schuepbach, D., Bermpohl, F., Walter, M., Ernst, J., Hell, D., Boeker, H., and Northoff, G. (2009a). Altered negative BOLD responses in the default-mode network during emotion processing in depressed subjects. Neuropsychopharmacology 34, 932-843.

Grimm, S., Ernst, J., Boesiger, P. Schuepbach, D., Hell, D., Boeker, H., and Northoff, G. (2009b). Increased self-focus in major depressive disorder is related to neural abnormalities in subcortical-cortical midline structures. Hum. Brain Mapp. 30, 2617-2627.

Hamilton, M. (1960). A rating scale for depression. J. Neurol. Neurosurg. Psychiatry 23, 56-62.

Hampson, M., Driesen, N. R., Skudlarski, P., Gore, J. C., and Constable, R. T. (2006). Brain connectivity related to working memory performance. $J$. Neurosci. 26, 13338-13343.

Hasler, G., van der Veen, J. W., Tumonis, T., Meyers, N., Shen, J., and Drevets, W. C. (2007). Reduced prefrontal glutamate/glutamine and gammaaminobutyric acid levels in major depression determined using proton magnetic resonance spectroscopy. Arch. Gen. Psychiatry 64, 193-200. 
Jafri, M. J., Pearlson, G. D., Stevens, M., and Calhoun, V. D. (2008). A method for functional network connectivity among spatially independent restingstate components in schizophrenia. Neuroimage 39, 1666-1681.

Kugaya, A., and Sanacora, G. (2005). Beyond monoamines: glutamatergic function in mood disorders. CNS Spectr. 10, 808-819.

Lerch, J.P., and Evans, A.C. (2005). Cortical thickness analysis examined through power analysis and a population simulation. Neuroimage 24, 163-173.

Lozano, A. M., Mayberg, H. S., Giacobbe, P., Hamani, C., Craddock, R. C., and Kennedy, S.H. (2008). Subcallosal cingulate gyrus deep brain stimulation for treatment-resistant depression. Biol. Psychiatry 64, 461-467.

MacQueen, G. M. (2009). Magnetic resonance imaging and prediction of outcome in patients with major depressive disorder. J. Psychiatry Neurosci. 34, 343-349.

Manji, H. K., Drevets, W. C., and Charney, D.S. (2001). The cellular neurobiology of depression. Nat. Med. 7, 541-547.

Margulies, D. S., Kelly, A. M. C., Uddin, L. Q., Biswal, B. B., Castellanos, F. X., and Milham, M. P. (2007). Mapping the functional connectivity of anterior cingulate cortex. Neuroimage 37, 579-588.

Mayberg, H. S. (1997). Limbic-cortical dysregulation: a proposed model of depression. J. Neuropsychiatry Clin. Neurosci. 9, 471-481.

Mayberg, H. S., Lozano, A. M., Voon, V., McNeely, H. E., Seminowicz, D., Hamani, C., Schwalb, J. M., and Kennedy, S. H. (2005). Deep brain stimulation for treatment-resistant depression. Neuron 45, 651-660.

Mennes, M., Kelly, C., Zuo, X.-N., Di Martino, A., Biswal, B., Castellanos, F. X., and Milham, M. P. (2010). Interindividual differences in resting state functional connectivity predict taskinduced BOLD activity. Neuroimage. 50, 1690-1701.

Menon, V., and Uddin,L.Q.(2010).Saliency, switching, attention, and control: a network model of insula function. Brain Struct. Funct. 214, 655-667.

Mesulam, M.M., and Mufson, E. J. (1982). Insula of the old world monkey. III: efferent cortical output and comments on function. J. Comp. Neurol. 212, 38-52.

Mufson, E. J., and Mesulam, M. M. (1982). Insula of the old world monkey. II: afferent cortical input and comments on the claustrum. J. Comp. Neurol. 212, 23-37.

Mullins, P.G., Chen,H.,Xu, J., Caprihan,A., and Gasparovic, C.(2008).Comparative reliability of proton spectroscopy techniques designed to improve detection of J-coupled metabolites. Magn. Reson. Med. 60, 964-969.
Murphy, K., Birn, R. M., Handwerker, D. A., Jones, T. B., and Bandettini, P. A. (2009). The impact of global signal regression on resting state correlations: are anti-correlated networks introduced? Neuroimage 44, 893-905.

Neumann, J., Fox, P. T., Turner, R., and Lohmann, G. (2010). Learning partially directed functional networks from meta-analysis imaging data. Neuroimage 49, 1372-1384.

Northoff, G., Walter, M., Schulte, R. F., Beck, J., Dydak, U., Henning, A., Boeker, H., Grimm, S., and Boesiger, P. (2007). GABA concentrations in the human anterior cingulate cortex predict negative BOLD responses in fMRI. Nat. Neurosci. 10, 1515-1517.

Ongür, D., Drevets, W. C., and Price, J. L. (1998). Glial reduction in the subgenual prefrontal cortex in mood disorders. Proc. Natl. Acad. Sci. U.S.A. 95, 13290-13295.

Provencher, S. W. (1993). Estimation of metabolite concentrations from localized in vivo proton NMR spectra. Magn. Reson. Med. 30, 672-679.

Raichle, M. E., and Mintun, M. A. (2006). Brain work and brain imaging. Annu. Rev. Neurosci. 29, 449-476.

Raichle, M. E., and Snyder, A. Z. (2007). A default mode of brain function: a brief history of an evolving idea. Neuroimage 37, 1083-1090; discussion 1097-1099.

Rajkowska, G., and Miguel-Hidalgo, J. J. (2007). Gliogenesis and glial pathology in depression. CNS Neurol. Disord. Drug Targets 6, 219-233.

Rajkowska, G., Miguel-Hidalgo, J. J., Wei, J., Dilley, G., Pittman, S. D., Meltzer, H. Y., Overholser, J. C., Roth, B. L., and Stockmeier,C.A.(1999).Morphometric evidence for neuronal and glial prefrontal cell pathology in major depression. Biol. Psychiatry 45, 1085-1098.

Rigucci, S., Serafini, G., Pompili, M., Kotzalidis, G. D., and Tatarelli, R. (2009). Anatomical and functional correlates in major depressive disorder: the contribution of neuroimaging studies. World J. Biol. Psychiatry, $1-16$.

Rothman, D. L., Behar, K. L., Hyder, F., and Shulman, R. G. (2003). In vivo NMR studies of the glutamate neurotransmitter flux and neuroenergetics: implications for brain function. Annu. Rev. Physiol. 65, 401-427.

Rowland, L. M., Bustillo, J. R., Mullins, P. G., Jung, R. E., Lenroot, R., Landgraf, E., Barrow, R., Yeo, R., Lauriello, J., and Brooks, W. M. (2005). Effects of ketamine on anterior cingulate glutamate metabolism in healthy humans: a 4-T proton MRS study. Am. J. Psychiatry 162, 394-396.

Salvadore, G., Cornwell, B. R., ColonRosario, V., Coppola, R., Grillon, C., Zarate, C. A., and Manji, H. K. (2009).
Increased anterior cingulate cortical activity in response to fearful faces: a neurophysiological biomarker that predicts rapid antidepressant response to ketamine. Biol. Psychiatry 65, 289-295.

Schubert, F., Gallinat, J., Seifert, F., and Rinneberg, H. (2004). Glutamate concentrations in human brain using single voxel proton magnetic resonance spectroscopy at 3 Tesla. Neuroimage 21, 1762-1771.

Seeley, W. W., Menon, V., Schatzberg, A. F., Keller, J., Glover, G. H., Kenna, H., Reiss, A. L., and Greicius, M. D. (2007). Dissociable intrinsic connectivity networks for salience processing and executive control. J. Neurosci. 27, 2349-2356.

Sheehan, D. V., Lecrubier, Y., Sheehan, K. H., Amorim, P., Janavs, J., Weiller, E. Hergueta, T., Baker, R., and Dunbar, G. C. (1998). The mini-international neuropsychiatric interview (MINI): the development and validation of a structured diagnostic psychiatric interview for DSM-IV and ICD-10. J. Clin. Psychiatry 59(Suppl. 20), 22-33; quiz 34-57.

Sheline, Y. I., Barch, D. M., Price, J. L. Rundle, M.M., Vaishnavi, S. N., Snyder, A.Z., Mintun, M.A., Wang, S., Coalson, R. S., and Raichle, M. E. (2009). The default mode network and self-referential processes in depression. Proc. Natl. Acad. Sci. U.S.A. 106, 1942-1947.

Shulman, R. G., Rothman, D. L., and Hyder, F. (2007). A BOLD search for baseline. Neuroimage 36, 277-281.

Sridharan, D., Levitin, D. J., and Menon, V. (2008). A critical role for the right fronto-insular cortex in switching between central-executive and defaultmode networks. Proc. Natl. Acad. Sci. U.S.A. 105, 12569-12574.

Uddin, L. Q., Kelly, A. M., Biswal, B. B. Xavier Castellanos, F., and Milham, M. P. (2009). Functional connectivity of default mode network components: correlation, anticorrelation, and causality. Hum. Brain Mapp. 30, 625-637.

Uddin, L. Q., and Menon, V. (2009). The anterior insula in autism: underconnected and under-examined. Neurosci. Biobehav. Rev. 33, 1198-1203.

Walter,M., Henning,A., Grimm, S., Schulte, R. F., Beck, J., Dydak, U., Schnepf, B., Boeker, H., Boesiger, P., and Northoff, G. (2009). The relationship between aberrant neuronal activation in the pregenual anterior cingulate, altered glutamatergic metabolism, and anhedonia in major depression. Arch. Gen. Psychiatry 66, 478-486.

Weissenbacher, A., Kasess, C., Gerstl, F., Lanzenberger, R., Moser, E., and Windischberger, C. (2009). Correlations and anticorrelations in resting-state functional connectivity MRI: a quantitative comparison of preprocessing strategies. Neuroimage 47, 1408-1416.
Whitfield-Gabrieli, S., Thermenos, H.W., Milanovic, S., Tsuang, M. T., Faraone, S. V., McCarley, R. W., Shenton, M. E., Green, A. I., Nieto-Castanon, A., LaViolette, P., Wojcik, J., Gabrieli, J. D. E., and Seidman, L. J. (2009). Hyperactivity and hyperconnectivity of the default network in schizophrenia and in first-degree relatives of persons with schizophrenia. Proc. Natl. Acad. Sci. U.S.A. 106, 1279-1284.

WHO. (1992). The ICD-10 Classification of Mental and Behavioral Disorders. Clinical Descriptions and Diagnostic Guidelines. Geneva: World Health Organization.

Yan, C.-G., and Zang, Y.-F. (2010). DPARSF: a MATLAB toolbox for "pipeline" data analysis of restingstate fMRI. Front. Syst. Neurosci. 4, 13. doi:10.3389/fnsys.2010.00013.

Yildiz-Yesiloglu, A., and Ankerst, D. P. (2006). Review of $1 \mathrm{H}$ magnetic resonance spectroscopy findings in major depressive disorder: a meta-analysis. Psychiatry Res. 147, 1-25.

Zarate, C. A., Singh, J. B., Carlson, P. J., Brutsche,N.E.,Ameli,R.,Luckenbaugh, D. A., Charney, D. S., and Manji, H. K. (2006). A randomized trial of an $\mathrm{N}$-methyl-D-aspartate antagonist in treatment-resistant major depression. Arch. Gen. Psychiatry 63, 856-864.

Zhou, Y., Yu, C., Zheng, H., Liu, Y., Song, M., Qin, W., Li, K., and Jiang, T. (2010). Increased neural resources recruitment in the intrinsic organization in major depression. J. Affect Disord. 121, 220-230.

Conflict of Interest Statement: The authors declare that the research was conducted in the absence of any commercial or financial relationships that could be construed as a potential conflict of interest.

Received: 05 February 2010; paper pending published: 10 March 2010; accepted: 18 June 2010; published online: 15 July 2010. Citation: Horn DI, Yu C, Steiner J, Buchmann J, Kaufmann J, Osoba A, Eckert U, Zierhut KC, Schiltz K, He H, Biswal B, Bogerts B and Walter M (2010) Glutamatergic and resting-state functional connectivity correlates of severity in major depression - the role of pregenual anterior cingulate cortex and anterior insula. Front. Syst. Neurosci. 4:33. doi: 10.3389/ fnsys.2010.00033

Copyright (c) 2010 Horn, Yu, Steiner, Buchmann, Kaufmann, Osoba, Eckert, Zierhut, Schiltz, He, Biswal, Bogerts and Walter. This is an open-access article subject to an exclusive license agreement between the authors and the Frontiers Research Foundation, which permits unrestricted use, distribution, and reproduction in any medium, provided the original authors and source are credited. 